\title{
Targeting YesI Associated Transcriptional Regulator Inhibits Hepatocellular Carcinoma Progression and Improves Sensitivity to Sorafenib: An in vitro and in vivo Study
}

This article was published in the following Dove Press journal: OncoTargets and Therapy

\author{
Liwen Guo* \\ Jiaping Zheng* \\ Jun Luo \\ Zhewei Zhang \\ Guoliang Shao \\ IDepartment of Interventional Radiology, \\ The Cancer Hospital of the University of \\ Chinese Academy of Sciences (Zhejiang \\ Cancer Hospital), Institute of Basic \\ Medicine and Cancer (IBMC), Chinese \\ Academy of Sciences, Hangzhou, \\ Zhejiang, 310022, People's Republic of \\ China
}

*These authors contributed equally to this work
Correspondence: Guoliang Shao

Department of Interventional Radiology,

The Cancer Hospital of the University of

Chinese Academy of Sciences (Zhejiang

Cancer Hospital), Institute of Basic

Medicine and Cancer (IBMC), Chinese

Academy of Sciences, I East Banshan

Road, Hangzhou 310022, People's

Republic of China

Tel +86-057l-88I 22222

Email shaogl@zjcc.org.cn
Purpose: The aim of this study was to investigate the role of Yes1 associated transcriptional regulator (YAP1) in the pathology of hepatocellular carcinoma (HCC) and its potential as a therapeutic target.

Methods: YAP1 expression in HCC and adjacent tissues was determined via immunohistochemistry; in HCC and human normal liver cell lines, expression was examined via Western blotting. The effects of YAP1 knockdown and overexpression were detected following transfection of HCC cells with siRNA-YAP1 recombinants or pcDNA3.1-YAP1 plasmids. A tumor xenograft model was constructed by implanting YAP1-knockdown lentivirus-infected Hep-3B cells into nude mice, and the animals were treated with sorafenib.

Results: In patients with HCC, YAP1 was upregulated in tumor tissue compared with adjacent tissue, and its high expression in the tumor was associated with increased Edmonson grade. In vitro, YAP1 expression was increased in Hep-3B, SK-HEP-1 and Huh7 cells, while it was similar in SMMC-7721 cells and LO2 cells. Meanwhile, YAP1 increased cell proliferation and invasion, promoted the progression of epithelial-mesenchymal transition, and inhibited cell apoptosis in HCC cells; furthermore, YAP1 knockdown combined with the administration of sorafenib decreased cell viability and increased cell apoptosis compared with YAP1 knockdown or treatment with sorafenib alone. In vivo, YAP1 knockdown inhibited tumor growth and metastasis, whereas it promoted apoptosis; meanwhile, YAP1 knockdown synergized with sorafenib to suppress tumor progression in HCC mice.

Conclusion: YAP1 is upregulated in both HCC tumor tissues and cell lines. Moreover, it promotes cell proliferation and invasion and promoted the progression of epithelialmesenchymal transition in vitro. Furthermore, targeting YAP1 inhibits HCC progression and improves sensitivity to sorafenib in vitro and in vivo.

Keywords: Yes1 associated transcriptional regulator, hepatocellular carcinoma, sorafenib, proliferation, invasion, apoptosis

\section{Introduction}

Hepatocellular carcinoma (HCC) represents the fifth most prevalent type of tumors and the second most common cause of cancer-related mortality globally; its incidence presents a progressive increment with age in all populations. ${ }^{1,2}$ The potential curative treatments include liver resection, orthotopic liver transplantation, radiofrequency ablation, and microwave ablation, while palliative treatment options 
include intra-arterial administration of chemotherapy, embolizing material, and radioactive particles. These treatment modalities have improved the clinical outcomes of a small portion of patients with HCC; however, the majority of patients continue to be associated with an unfavorable prognosis owing to the high rate of tumor recurrence and increasing drug resistance. ${ }^{3-5}$ Thus, knowledge on the molecular biology of HCC is essential for the selection of the most appropriate treatment and improvement of the clinical outcomes in HCC management.

Yes1 associated transcriptional regulator (YAP1) is an important regulator of the Hippo pathway, which is an essential tumor-regulating signaling pathway in various malignancies. ${ }^{6,7}$ Recent studies indicated that YAP1 is a candidate oncogene and is associated with tumorigenesis via diverse biological processes, including the promotion of malignant phenotypes, regulation of epithelialmesenchymal transition (EMT), and expansion of cancer stem cells. ${ }^{8,9}$ For example, in breast cancer, YAP1 promotes tumor invasiveness by regulating the phosphorylation of focal adhesion kinase. Its knockdown sensitizes tumor cells to radiation by inhibiting epidermal growth factor receptor (EGFR)/PI3K/AKT signaling axis and increasing DNA damage. ${ }^{9,10}$ In another study, YAP1 was upregulated in gastric cancer tissues compared with noncancerous paired normal tissues, while its knockdown attenuated the proliferation of cancer cells and enhanced sensitivity to cisplatin by targeting the EGFR. ${ }^{11}$ According to the previous studies, we hypothesized that YAP1 may be implicated in the development and progression of HCC, and may serve as a target in the treatment of $\mathrm{HCC}$, in combination with other drugs; however, the currently available evidence is insufficient. Therefore, in the present study, we explored the association of YAP1 with clinicopathological features of patients with $\mathrm{HCC}$, and further investigated its role in regulating cell proliferation, apoptosis, invasion, and expression of its downstream gene (Tead) and EMT markers in vitro, as well as the synergistic effect of YAP1 knockdown with sorafenib in vivo in this setting.

\section{Materials and Methods}

\section{Specimens and Cell Lines}

Seventy HCC biopsy specimens and 20 paired adjacent tissue specimens were purchased from Shanghai Outdo Biotech Co., Ltd. (Shanghai, China). The usage of these specimens was approved by the Institutional Review
Board of the company, and informed consent was provided by the patients prior to specimen collection. The human HCC cell lines Hep-3B, SMMC-7721, SK-HEP-1, and Huh-7, as well as the human normal liver cell line LO2, were purchased from the American Type Culture Collection (Manassas, VA, USA) and cultured in 90\% Dulbecco's modified Eagle's medium (Gibco, NY, USA) supplemented with $10 \%$ fetal bovine serum (Gibco), 100 $\mathrm{U} / \mathrm{mL}$ of penicillin, and $100 \mu \mathrm{g} / \mathrm{mL}$ of streptomycin. All cell lines were maintained in a humidified incubator with an atmosphere of $5 \% \mathrm{CO}_{2}$ at $37^{\circ} \mathrm{C}$. Following cell culture, the expression of YAP1 protein in the cell lines was determined by Western blotting.

\section{Immunohistochemistry (IHC) Assay}

HCC specimens and adjacent tissue specimens were cut into $4-\mu \mathrm{m}$ paraffin-embedded sections, mounted on slides, deparaffinized with xylene and rehydrated with graded ethanol, and blocked with 3\% hydrogen peroxide. Heatinduced antigen retrieval was performed using a steamer at $95^{\circ} \mathrm{C}$. The primary antibody Rabbit Anti-YAP1 antibody (Abcam, MA, USA) was diluted at a ratio of 1:50. Incubation was performed at $4{ }^{\circ} \mathrm{C}$ overnight, followed by incubation with a secondary antibody Goat Anti-Rabbit IgG H\&L (horseradish peroxidase [HRP]) (Abcam) in 1:50,000 dilution at $37^{\circ} \mathrm{C}$ for $60 \mathrm{~min}$. Diaminobenzidine was subsequently applied for $10 \mathrm{~min}$. Finally, the sections were counterstained with hematoxylin, dehydrated, covered, and visualized. According to previous studies, ${ }^{12,13}$ the expression of YAP1 was determined by the density of positive cells and the intensity of staining. The density was scored based on the proportion of positive cells ( 0 : none; $1: \leq 10 \% ; 2: 11-25 \% ; 3: 26-50 \% ; 4:>50 \%$, and the intensity was scored based on the average staining intensity of positive cells ( 0 : none; 1 : weak; 2 : intermediate; 3 : strong). The final score was the product of the density score and intensity score, ranging $0-12$. Patients were classified into two groups: YAP1 low expression (score: 0-3) and YAP1 high expression (score: 4-12).

\section{Transfection}

Three small-interfering RNAs (siRNA) against YAP1 (siRNA-YAP1) and non-targeting siRNA (siRNA-NC) were designed and synthesized by GenePharma (Shanghai, China). A pcDNA3.1-YAP1 plasmid and the control plasmid (pcDNA3.1-NC) were also synthesized by GenePharma. Using Lipofectamine 2000 (Invitrogen, CA, USA) according to the instructions provided by the 
manufacturer, Hep-3B cells were transfected with siRNAYAP1 recombinants or siRNA-NC, as well as with the pcDNA3.1-YAP1 plasmid or pcDNA3.1-NC plasmid. The SMMC-7721 cells were transfected with the pcDNA3.1-YAP1 plasmid or pcDNA3.1-NC plasmid. The non-transfected Hep-3B cells and SMMC-7721 cells served as controls check (CK). The expression of YAP1 protein in the cells was determined by Western blotting after $24 \mathrm{~h}, 48 \mathrm{~h}$, and $72 \mathrm{~h}$ of incubation.

\section{Western Blotting Assay}

Cells were lysed in radioimmunoprecipitation assay lysis buffer (Sigma-Aldrich, MO, USA). After electrophoresis on a $12 \%$ or $15 \%$ sodium dodecyl sulfate-polyacrylamide gel electrophoresis gel, proteins were transferred onto polyvinylidene difluoride membranes. Subsequently, the membranes were blocked with 5\% non-fat milk and incubated overnight at $4^{\circ} \mathrm{C}$ with the following primary antibodies: Rabbit Anti-YAP1 antibody (1:5000 dilution; Abcam), Rabbit Anti-YAP1 (phosphorylated S127) (pYAP1) antibody (1:50000 dilution; Abcam), Rabbit Anti-Ki67 antibody (1:5,000 dilution; Abcam), Rabbit Anti-TEAD-1 antibody (1:50000 dilution; Abcam), Rabbit Anti-E-cadherin antibody (1:50000 dilution; Abcam), Rabbit Anti-N-cadherin antibody (1:1000 dilution, Abcam), and Rabbit Anti-glyceraldehyde-3-phosphate dehydrogenase (Anti-GAPDH) antibody (1:10000 dilution, Abcam). The HRP-conjugated secondary antibody Goat Anti-Rabbit IgG H\&L (HRP) (1:20000 dilution, Abcam, USA) was added and incubated at room temperature for $1 \mathrm{~h}$. Signals were visualized after chemiluminescence reaction with horseradish peroxidase substrate. The relative protein expressions of YAP1, pYAP1, Ki67, Tead, EMT markers (E-Cadherin and N-Cadherin) in different cells were normalized to the GAPDH concentration, and the quantitative analysis was performed using the Image $\mathbf{J}$ software (National Institutes of Health (NIH), USA).

\section{Cell Proliferation, Apoptosis, and Invasion Assays}

Cell proliferation was determined using the Cell Counting Kit-8 cell viability assay kit (Dojindo, Kyushu Japan). In brief, 5,000 cells per well were seeded in 96-well plates. At $0 \mathrm{~h}, 24 \mathrm{~h}, 48 \mathrm{~h}$ or $72 \mathrm{~h}$ after incubation, $10 \mu \mathrm{L}$ of cell viability assay kit solution was added to each well. Subsequently, the absorbance of each well was measured at $450 \mathrm{~nm}$ using a microplate reader (Biotek, VT, USA). Cell apoptosis was assessed by fluorescein isothiocyanate (FITC)-labeled annexin-V and propidium iodide (PI) (Sigma-Aldrich, USA) using flow cytometry. Briefly, following transfection for $48 \mathrm{~h}$, the cells were collected and stained in $500 \mu \mathrm{L}$ of a solution containing Annexin V-FITC in the dark. Then, PI was added for 5 min in the dark. Flow cytometry (Becton Dickinson, NJ, USA) was used to detect the fluorescent signal in the cells. Cell invasion ability was assessed at $48 \mathrm{~h}$ after transfection through the Transwell assay using invasion chambers (BD Biosciences, CA, USA), as previously described. ${ }^{14}$ The invading cells that adhered to the lower surface of the membrane were viewed through an optical microscope (Nikon, Tokyo, Japan) and counted by the ImageJ software (National Institutes of Health).

\section{Lentivirus Infection and Assessment of Drug-Sensitivity}

The YAP1-knockdown lentivirus (YAP1-KD-LV) and negative control lentivirus (NC-LV) were synthesized by GenePharma and used to infect Hep-3B cells. The cells infected with the YAP1-knockdown lentivirus were defined as YAP1-KD-LV cells; those infected with negative control lentivirus were defined as control cells; cells treated with $30 \mathrm{mg} / \mathrm{kg}$ sorafenib were defined as sorafenib cells; and those infected with YAP1-knockdown lentivirus and treated with sorafenib were defined as sorafenib and YAP1-KD-LV cells. As shown before, cell viability was determined using the Cell Counting Kit-8 cell viability assay kit (Dojindo) and cell apoptosis was assessed by FITC-labeled annexin-V and PI (Sigma-Aldrich) using flow cytometry.

\section{Construction of a Tumor Xenograft Model}

The animal study was approved by the Institutional Animal Care and Use Committee of Cancer Hospital of the University of Chinese Academy of Sciences (Hangzhou, China), following the Guidelines for the ethical review of laboratory animal welfare People's Republic of China National Standard GB/T35892-2018. ${ }^{15}$ Twenty BALB/c male nude mice (4-5 weeks old, 18-20 g) were purchased from Shanghai Lab. Animal Research Center (Shanghai, China) and housed in a standard animal laboratory with free access to food and water. Subsequently, stably infected Hep-3B cells were screened out using 
puromycin (Sigma-Aldrich). After the harvest, stably infected Hep-3B cells were suspended in serum-free DMEM $\left(3 \times 10^{6} / \mathrm{mL}\right)$. Subsequently, the YAP1-KD-LV infected Hep-3B cells or NC-LV infected Hep-3B cells were injected into the upper flank region of nude mice (200 $\mu \mathrm{L}$ per animal) for construction of the tumor xenograft model. After 7 days, the mice were assigned into four groups (five mice per group) and treated as follows: (1) Control group: mice (implanted with NC-LV-infected Hep$3 \mathrm{~B}$ cells) were treated with saline solution by oral gavage once daily for 30 days; (2) Sorafenib group: mice (implanted with NC-LV-infected Hep-3B cells) were treated with $30 \mathrm{mg} / \mathrm{kg}$ sorafenib (diluted in saline solution) by oral gavage once daily for 30 days; (3) YAP1-KD-LV group: mice (implanted with YAP1-KD-LV-infected Hep$3 \mathrm{~B}$ cells) were treated with saline solution by oral gavage once daily for 30 days; and (4) Sorafenib \& YAP1-KD-LV group: mice (implanted with YAP1-KD-LV-infected Hep$3 \mathrm{~B}$ cells) were treated with $30 \mathrm{mg} / \mathrm{kg}$ sorafenib (diluted in saline solution) by oral gavage once daily for 30 days. After 30 days of treatment, all mice were sacrificed with pentobarbital, and the tumors were resected and weighed. Furthermore, tumor specimens were formalin-fixed and paraffin-embedded. The cell apoptosis in the tumor specimens was detected by the terminal deoxynucleotidyl transferase-mediated dUTP nick end labelling (TUNEL) assay using the TumorTACS in situ Apoptosis Detection kit (R\&D, MN, USA) according to the instructions provided by the manufacturer. The expression of YAP1, matrix metalloproteinase 3 (MMP3), and MMP9 in the specimens was assessed by IHC assay. The primary antibodies (all purchased from Abcam) were: Rabbit Anti-YAP1 antibody (1:5,000 dilution); Rabbit Anti-MMP3 antibody (1:50 dilution); and Rabbit Anti-MMP9 antibody (1:1,000 dilution). The secondary antibody (also purchased from Abcam) was Goat Anti-Rabbit IgG H\&L (HRP) (1:20,000 dilution).

\section{Statistical Analysis}

Data were expressed as the mean \pm standard deviation or number (percentage). In the clinical data analyses, the comparison of YAP1 expression between tumor tissue and adjacent tissue was determined using the McNemar test. The comparison of clinicopathological features between patients with low and high expression of YAP1 was conducted using the chi-squared test. In the experiment-related analyses, the comparison among groups was determined through one-way analysis of variance, followed by Dunnett's multiple comparisons test. Moreover, the comparison between two groups was performed using the unpaired $t$-test. Furthermore, the degree of drug interaction was calculated via calculation of the combination index (CI) according to previous evidence: $\mathrm{CI}<1$ indicated a synergistic effect of drugs; $\mathrm{CI}=1$ indicated an additive effect; and $\mathrm{CI}>1$ indicated antagonism. ${ }^{16}$ All statistical analyses were performed using GraphPad Prism 7.01 (GraphPad Software, Inc., La Jolla, CA, USA) and SPSS 22.0 (IBM, Corp., Armonk, NY, USA) software. $P<0.05$ was considered significant, and the $P$ value was displayed as $* P<0.05,{ }^{* *} P<0.01,{ }^{* * *} P<0.001$, and NS $(P>$ $0.05)$ in the figures related to the experiments.

\section{Results \\ YAPI Expression in HCC Tumor Tissue and Adjacent Tissue}

Representative images of YAP1 low expression in adjacent tissue and YAP1 high expression in tumor tissue were exhibited (Figure 1A). The comparison of the percentage of YAP1 low/high expression between tumor tissue and adjacent tissue indicated that YAP1 was upregulated in HCC tumor tissue compared with adjacent tissue $(P<0.001)$ (Figure 1B).

\section{Correlation of Tumor YAPI Expression in Tumors with Clinicopathological Features in Patients with HCC}

High expression of YAP1 in tumors was associated with increased Edmonson grade ( $P=0.023$ ), however, there was no correlation of tumor YAP1 expression in tumors with age ( $P=0.940)$, sex $(P=0.289)$, tumor size $(P=0.638)$, TNM stage ( $P=0.717)$, vascular invasion $(P=0.289)$, adjacent organ invasion $(P=0.709)$, or number of tumor nodules $(P=0.518)$ (Table 1). Detailed information on the clinicopathological features of patients with $\mathrm{HCC}$ is provided in Table 1.

\section{YAPI and pYAPI Expression in HCC Cell Lines and Normal Cells}

YAP1 expression was higher in Hep-3B $(\mathrm{P}<0.001)$, SKHEP-1 $(\mathrm{P}<0.01)$, and Huh7 $(\mathrm{P}<0.01)$ cells, but exhibited a similar trend in SMMC-7721 $(\mathrm{P}>0.05)$ cells compared with LO2 cells (Figure 2A and B). pYAP1 expression was lower in Hep-3B $(\mathrm{P}<0.001)$, SK-HEP-1 $(\mathrm{P}<0.001)$, and Huh7 cells $(\mathrm{P}<0.01)$, but presented a similar trend in SMMC-7721 cells $(\mathrm{P}>0.05)$ compared with LO2 cells. 
A

YAP1 low in adjacent tissue YAP1 high in tumor tissue
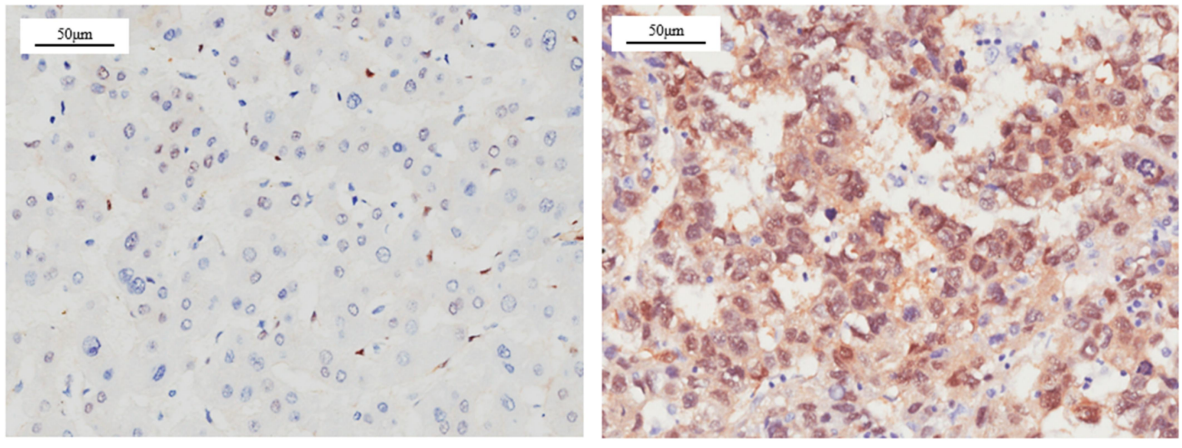

B

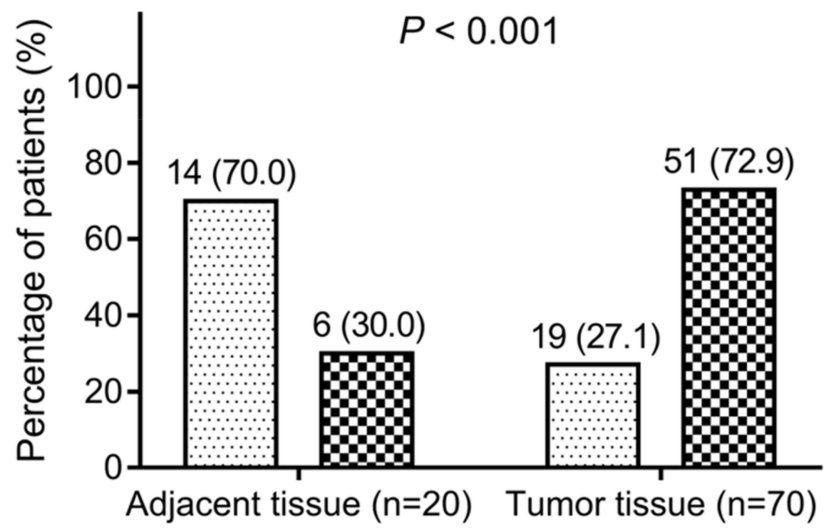

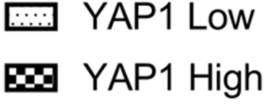

$\infty$ YAP1 High

Figure I YAPI was upregulated in HCC tumor tissues compared with adjacent tissues. Representative images of low YAPI expression in adjacent tissue and high YAPI expression in tumor tissue (A). Comparison of YAPI expression between adjacent tissue and tumor tissue (B).

Abbreviations: YAPI, YesI associated transcriptional regulator; HCC, hepatocellular carcinoma.

Hep-3B and SMMC-7721 cells were selected for the following cellular experiments.

\section{YAPI and pYAPI Expressions After Transfection of Hep-3B and SMMC-772I \\ Cells}

Hep-3B cells were transfected with three types of siRNAYAP1 recombinants and siRNA-NC. The expression of YAP1 in cells transfected with siRNA-YAP1 recombinants was decreased compared with that noted in cells transfected with siRNA-NC at $48 \mathrm{~h} \quad(P<0.001)$ and 72 $\mathrm{h}(P<0.001)$ (Figure $3 \mathrm{~A}$ and $\mathrm{B})$. Considering the greatest effect of the third siRNA-YAP1 recombinant in suppressing YAP1, it was selected for the following cellular function experiments. SMMC-7721 cells were transfected with the pcDNA3.1-YAP1 plasmid or pcDNA3.1-NC plasmid. YAP1 expression in cells transfected with the pcDNA3.1-YAP1 plasmid was increased compared with that recorded in cells transfected with the pcDNA3.1-NC plasmid at $24 \mathrm{~h}(P<0.001), 48 \mathrm{~h}(P<0.01)$, and 72 $\mathrm{h}(P<0.01)$ (Figure $3 \mathrm{C}$ and $\mathrm{D})$. These data indicated the successful transfection.

\section{Effect of YAPI Knockdown and Overexpression on the Proliferation of HCC Cells}

In Hep-3B cells, proliferation in cells transfected with siRNAYAP1 recombinants was decreased compared with that observed in cells transfected with siRNA-NC at 48 h $(P<0.05)$ and $72 \mathrm{~h}(P<0.05)$ (Figure $4 \mathrm{~A})$; furthermore, Ki67 protein expression was decreased in siRNA-YAP1 recombinants transfected cells compared with siRNA-NC transfected cells $(P<0.05)$ (Figure 4B and $C)$. In SMMC7721 cells, proliferation in cells transfected with the pcDNA3.1-YAP1 plasmid was increased compared with that detected in cells transfected with the pcDNA3.1-NC plasmid at 
Table I Association of Tumor YAPI Expression with Clinicopathological Features in HCC Patients

\begin{tabular}{|c|c|c|c|c|}
\hline \multirow[t]{2}{*}{ Category } & \multirow{2}{*}{$\begin{array}{l}\text { All } \\
\text { Cases } \\
(\mathbf{N}=70)\end{array}$} & \multicolumn{2}{|l|}{ YAPI } & \multirow[t]{2}{*}{$P$-value } \\
\hline & & $\begin{array}{l}\text { High } \\
(n=5 I)\end{array}$ & $\begin{array}{l}\text { Low } \\
(n=19)\end{array}$ & \\
\hline $\begin{array}{l}\text { Age } \\
\qquad>45 \text { years }\end{array}$ & $\begin{array}{l}52 \\
(74.3)\end{array}$ & $38(74.5)$ & I4 (73.7) & 0.940 \\
\hline$\leq 45$ years & $\begin{array}{l}18 \\
(25.7)\end{array}$ & $13(25.5)$ & $5(26.3)$ & \\
\hline Sex & & & & 0.289 \\
\hline Men & $\begin{array}{l}66 \\
(94.3)\end{array}$ & 49 (96.I) & 17 (89.5) & \\
\hline Women & $4(5.7)$ & $2(3.9)$ & $2(10.5)$ & \\
\hline $\begin{array}{l}\text { Edmonson grade } \\
\qquad \text { III }\end{array}$ & $\begin{array}{l}48 \\
(68.6)\end{array}$ & 31 (60.8) & 17 (89.5) & 0.023 \\
\hline III-IV & $\begin{array}{l}22 \\
(31.4)\end{array}$ & $20(39.2)$ & $2(10.5)$ & \\
\hline $\begin{array}{l}\text { Tumor size } \\
\quad \leq 5 \mathrm{~cm}\end{array}$ & $\begin{array}{l}33 \\
(47.1)\end{array}$ & $25(49.0)$ & $8(42.1)$ & 0.638 \\
\hline$>5 \mathrm{~cm}$ & $\begin{array}{l}27 \\
(38.6)\end{array}$ & $19(37.3)$ & $8(42.1)$ & \\
\hline Unknow & $\begin{array}{l}10 \\
(14.3)\end{array}$ & - & - & \\
\hline $\begin{array}{l}\text { TNM stage } \\
\text { I-II }\end{array}$ & $\begin{array}{l}10 \\
(14.3)\end{array}$ & $8(15.7)$ & $2(10.5)$ & 0.717 \\
\hline III-IV & $\begin{array}{l}60 \\
(85.7)\end{array}$ & $43(84.3)$ & 17 (89.5) & \\
\hline $\begin{array}{l}\text { Vascular invasion } \\
\text { Present }\end{array}$ & $\begin{array}{l}54 \\
(77.1)\end{array}$ & $4 \mid(80.4)$ & $13(68.4)$ & 0.289 \\
\hline Absent & $\begin{array}{l}16 \\
(22.9)\end{array}$ & $10(19.6)$ & $6(31.6)$ & \\
\hline $\begin{array}{l}\text { Adjacent organ } \\
\text { invasion } \\
\text { Present } \\
\text { Absent }\end{array}$ & $\begin{array}{l}5(7.1) \\
65 \\
(92.9)\end{array}$ & $\begin{array}{l}4(7.8) \\
47(92.2)\end{array}$ & $\begin{array}{l}\text { I (5.3) } \\
18(94.7)\end{array}$ & 0.709 \\
\hline $\begin{array}{l}\text { Numbers of tumor } \\
\text { nodules }\end{array}$ & & & & 0.518 \\
\hline I & $\begin{array}{l}44 \\
(62.9)\end{array}$ & $30(58.8)$ & 14 (73.7) & \\
\hline$\geq 2$ & $\begin{array}{l}16 \\
(22.9)\end{array}$ & I3 (25.5) & $3(15.8)$ & \\
\hline Unknow & $\begin{array}{l}10 \\
(14.3)\end{array}$ & - & - & \\
\hline
\end{tabular}

Abbreviation: HCC, hepatocellular carcinoma.
A
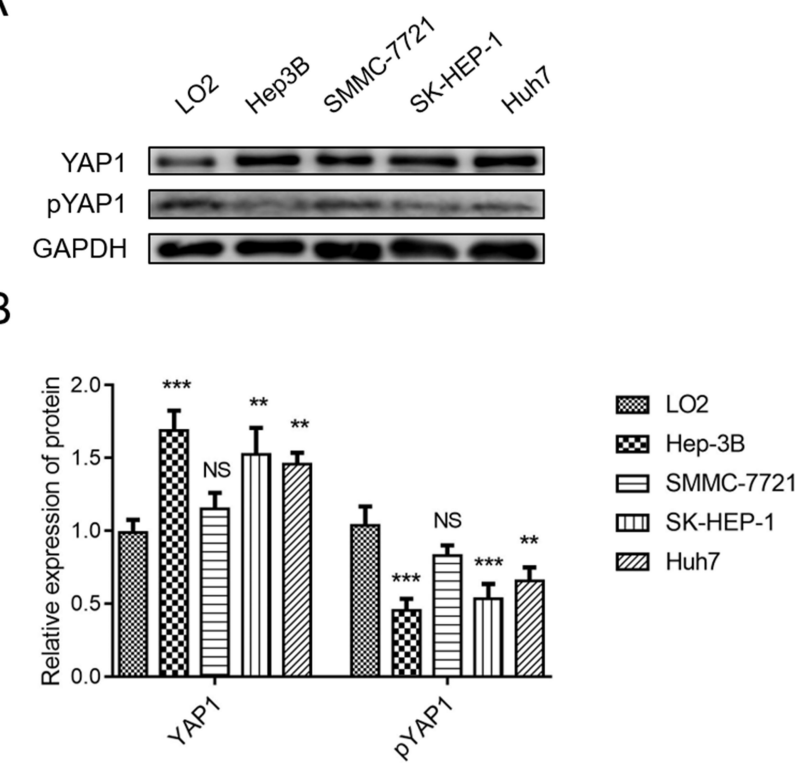

Figure 2 YAPI was upregulated, whereas PYAPI was downregulated, in HCC cell lines compared with normal liver cells. Comparison of YAPI and PYAPI expression between human HCC cell lines (Hep-3B, SMMC-772I, SK-HEP-I, Huh-7) and a human normal liver cell line (LO2) (A and B). P value was displayed as **P < 0.01 , ***P $<0.001$, and NS $(\mathrm{P}>0.05)$.

Abbreviations: YAPI, YesI associated transcriptional regulator; $\mathrm{HCC}$, hepatocellular carcinoma; PYAPI, phosphorylated YAPI; CK, control check.

$48 \mathrm{~h}(P<0.05)$ and $72 \mathrm{~h}(P<0.05)$ (Figure 4D); Meanwhile, Ki67 protein expression in cells transfected with the pcDNA3.1-YAP1 plasmid was higher than that determined in cells transfected with the pcDNA3.1-NC plasmid $(\mathrm{P}<0.05)$ (Figure 4E and F).

\section{Effect of YAPI Knockdown and Overexpression on Apoptosis in HCC Cells}

In Hep-3B cells, the cell apoptosis rate in cells transfected with the siRNA-YAP1 recombinants was increased versus that observed in cells transfected with siRNA-NC $(P<0.001)$ (Figure 5A and B). In SMMC-7721 cells, the cells transfected with the pcDNA3.1-YAP1 plasmid exhibited a declining trend in cell apoptosis rate versus those transfected with the pcDNA3.1-NC plasmid, though the difference was not statistically significant $(\mathrm{P}>0.05)$ (Figure $5 \mathrm{C}$ and $\mathrm{D}$ ).

\section{Effect of YAPI Knockdown and}

\section{Overexpression on Cell Invasion in HCC Cells}

In Hep-3B cells, the number of invasive cells was decreased in cells transfected with siRNA-YAP1 recombinants 
Hep-3B cells

A

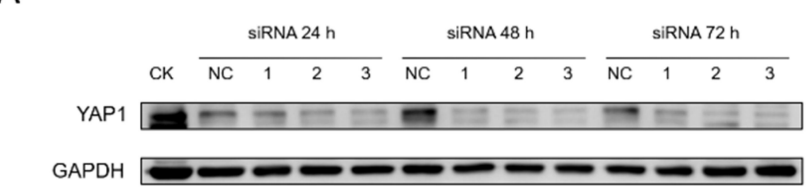

B



SMMC-7721 cells

C

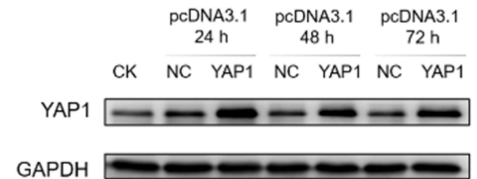

D

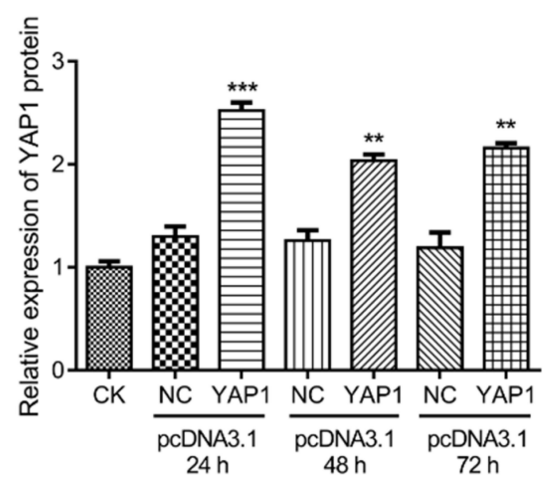

Figure 3 Establishment of YAPI knockdown and overexpression in HCC cells. Comparison of YAPI expression between cells transfected with siRNA-YAPI recombinants and those transfected with siRNA-NC (HEP-3B cells) (A and B). Comparison of YAPI expression between cells transfected with the pcDNA3.I-YAPI plasmid and those transfected with the pcDNA3.I-NC plasmid (SMMC-772I cells) $(\mathbf{C}$ and D). P value was displayed as $* * \mathrm{P}<0.01, * * * \mathrm{P}<0.00 \mathrm{I}$, and NS (P>0.05).

Abbreviations: YAPI, YesI associated transcriptional regulator; HCC, hepatocellular carcinoma; siRNA, small-interfering RNAs; NC, negative control; CK, control check.

compared with that recorded in cells transfected with siRNANC $(P<0.01)$ (Figure 6A and B). In SMMC-7721 cells, the number of invasive cells was increased in cells transfected with the pcDNA3.1-YAP1 plasmid compared with that determined in cells transfected with the pcDNA3.1-NC plasmid $(P<0.001)$ (Figure 6C and D).

\section{Effect of YAPI Knockdown and Overexpression on the Expression of Tead and EMT Markers in HCC Cells}

In Hep-3B cells, the expression of Tead $(\mathrm{P}<0.05)$ and N-cadherin $(\mathrm{P}<0.05)$ was decreased, whereas that of E-cadherin $(\mathrm{P}<0.05)$ was increased in cells transfected with siRNA-YAP1 recombinants versus cells transfected with siRNA-NC (Figure 7A and B). In SMMC-7721 cells, the expression of Tead $(\mathrm{P}<0.01)$ and $\mathrm{N}$-cadherin $(\mathrm{P}<0.01)$ was higher, whereas that of E-cadherin $(\mathrm{P}<0.05)$ was lower in cells transfected with the pcDNA3.1-YAP1 plasmid versus those transfected with the pcDNA3.1-NC plasmid (Figure 7C and D).

\section{Validation of the Effect of YAPI} Overexpression on the Proliferation, Apoptosis, and Invasion of Hep-3B Cells Transfection of Hep-3B cells with the pcDNA3.1-YAP1 plasmid or pcDNA3.1-NC plasmid led to an increase in YAP1 protein expression in the former group of cells $(\mathrm{P}<0.001)$ (Figure 8A and $\mathrm{B})$. In Hep-3B cells, cell proliferation (optical density $450 \mathrm{~nm}$ absorbance) was higher at $48 \mathrm{~h}$ and $72 \mathrm{~h}$ (both $\mathrm{P}<0.05$ ) (Figure 8C); Ki67 protein expression was higher $(\mathrm{P}<0.01)$ (Figure $8 \mathrm{D}$ and $\mathrm{E})$, cell apoptosis rate was lower $(P<0.05)$ (Figure $8 \mathrm{~F}$ and $\mathrm{G})$, and the number of invasive cells was elevated $(\mathrm{P}<0.05)$ (Figure $8 \mathrm{H}$ and $\mathrm{I}$ ) in cells transfected with the pcDNA3.1-YAP1 plasmid versus those transfected with the pcDNA3.1-NC plasmid. These data verified that YAP1 promoted the proliferation and invasion, whereas it inhibited apoptosis of HCC cells.

\section{Validation of the Effect of YAPI Overexpression on Tead and EMT Markers in Hep-3B Cells}

In Hep-3B cells, the expression of Tead $(\mathrm{P}<0.01)$ and $\mathrm{N}$-cadherin $(\mathrm{P}<0.01)$ was increased, whereas that of E-cadherin $(\mathrm{P}<0.01)$ was decreased in cells transfected with the pcDNA3.1-YAP1 plasmid versus cells transfected with the pcDNA3.1-NC plasmid (Figure 9A and B). These data supported that YAP1 increased the expression of Tead and promoted EMT progression in HCC cells. 
Hep-3B cells

A

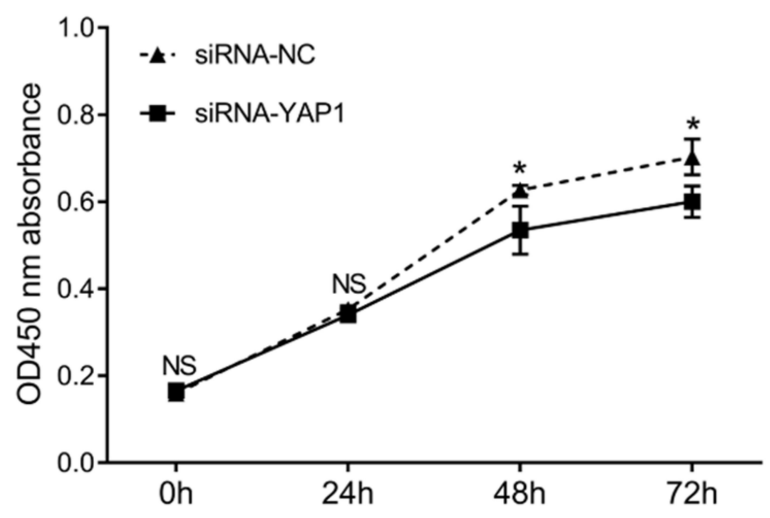

B

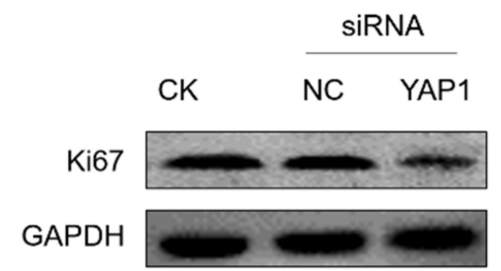

C

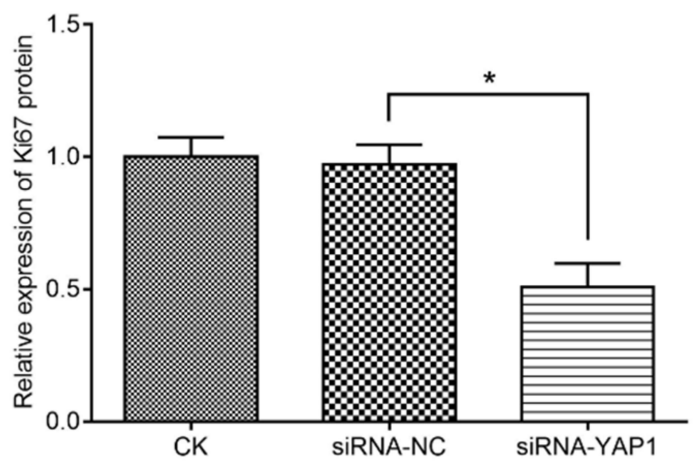

SMMC-7721 cells

D



E



F

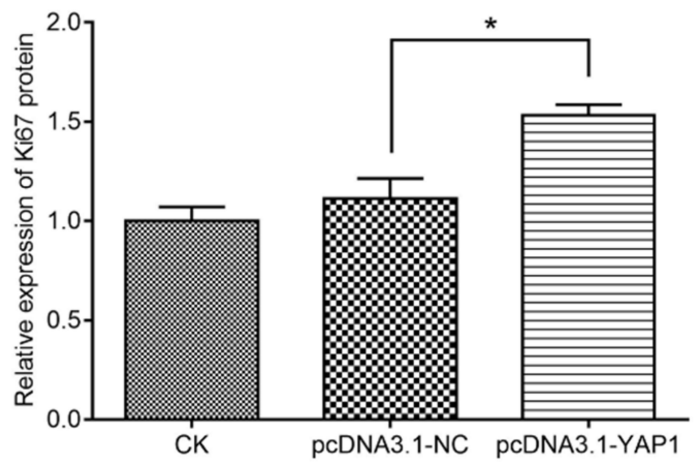

Figure 4 YAPI promoted proliferation in HCC cells. Comparison of OD450 nm absorbance by CCK-8 (A) and Ki67 protein expression (B and C) between Hep-3B cells transfected with siRNA-YAPI recombinants and those transfected with siRNA-NC at $24 \mathrm{~h}, 48 \mathrm{~h}$, and $72 \mathrm{~h}$ after transfection. Comparison of OD450 nm absorbance by CCK8 (D) and Ki67 protein expression (E and F) between SMMC-772I cells transfected with the pcDNA3.I-YAPI plasmid and those transfected with the pcDNA3.I-NC plasmid at $24 \mathrm{~h}, 48 \mathrm{~h}$, and $72 \mathrm{~h}$ after transfection. $P$ value was displayed as $* P<0.05$ and NS $(P>0.05)$.

Abbreviations: YAPI, YesI associated transcriptional regulator; HCC, hepatocellular carcinoma; siRNA, small-interfering RNAs; NC, negative control; OD, optical density; CCK-8, Cell Counting Kit-8; CK, control check.

\section{YAPI Knockdown and Its Synergistic Effect with Sorafenib in HCC Cells}

Cellular experiments were conducted in Hep-3B cells to investigate the combined effect of YAP1 knockdown and sorafenib in HCC cells. The relative cell viability was decreased in sorafenib cells $(\mathrm{P}<0.05)$ and YAP1-KD-LV cells $(\mathrm{P}<0.01)$ compared with control cells, and was further reduced in sorafenib and YAP1-KD-LV cells compared with sorafenib cells $(\mathrm{P}<0.01)$ and YAP1-KD-LV cells $(\mathrm{P}<0.05)$ (Figure 10A). Furthermore, the synergistic effect of YAP1 knockdown and sorafenib was assessed; the obtained CI value was $0.8825 \pm 0.2268$, implying 
Hep-3B cells

A

$A$
$\bar{\alpha}$



B

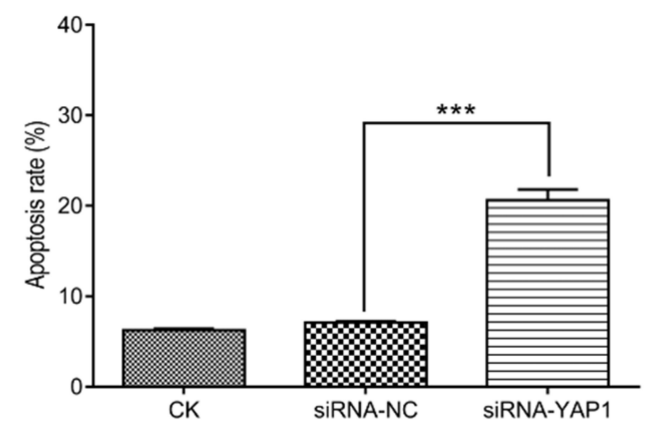

SMMC-7721 cells

C



D



Figure 5 YAPI decreased apoptosis in HCC cells. Comparison of the cell apoptosis rate by the AV/PI assay between Hep-3B cells transfected with siRNA-YAPI recombinants and those transfected with siRNA-NC at $48 \mathrm{~h}$ after transfection (Aand B). Comparison of the cell apoptosis rate by the AV/PI assay between SMMC-772I cells transfected with the pcDNA3. I-YAPI plasmid and those transfected with the pcDNA3. I-NC plasmid at $48 \mathrm{~h}$ after transfection (Cand D). P value was displayed as ***P $<0.001$, and NS $(P>0.05)$.

Abbreviations: YAPI, YesI associated transcriptional regulator; HCC, hepatocellular carcinoma; siRNA, small-interfering RNAs; NC, negative control; AV/PI, Annexin V/ Propidium lodide; CK, control check.

synergism. Moreover, the cell apoptosis rate in sorafenib cells $(\mathrm{P}<0.01)$ and YAP1-KD-LV cells $(\mathrm{P}<0.001)$ was increased versus that recorded in control cells. This rate was further enhanced in Sorafenib\&YAP1-KD-LV cells compared with sorafenib cells $(\mathrm{P}<0.001)$ and YAP1-KDLV cells $(\mathrm{P}<0.01)$ (Figure 10B and $\mathrm{C}$ ).

\section{YAPI Knockdown and Its Synergistic Effect with Sorafenib in Treating HCC Mice}

A tumor xenograft model was constructed by injecting Hep-3B cells infected with YAP1-KD-LV or NC-LV into nude mice. Tumor size in the Sorafenib\&YAP1-KD-LV group was smaller compared with those noted in the YAP1-KD-LV and Sorafenib groups. Moreover, it was smaller in the YAP1-KD-LV group compared with the Control group, and similar between the Sorafenib and Control groups (Figure 11A). In addition, the tumor weight was reduced in the Sorafenib \& YAP1-KD-LV group compared with the YAP1-KD-LV $(P<0.001)$ and Sorafenib groups $(P<0.001)$. It was also decreased in the YAP1-KD-LV group versus the Control group $(P<0.05)$, and was similar between the Sorafenib and Control groups $(P>0.05)$ (Figure 11B). Moreover, a CI value of 0.6801 \pm 0.2139 was obtained for the synergistic effect of YAP1 knockdown and sorafenib, suggesting synergism. Furthermore, the TUNEL assay indicated that the rate of tumor apoptosis in the Sorafenib \& YAP1-KD-LV group was increased compared with those recorded for the YAP1-KD-LV and Sorafenib groups; this rate was also elevated in the YAP1-KD-LV and Sorafenib groups versus the Control group (Figure 11C). In addition, the IHC assay indicated that YAP1-KD-LV decreased the expression of YAP1, MMP3, and MMP9; the combination of YAP1-KDLV and sorafenib further reduced the expression of YAP1, MMP3, and MMP9 (Figure 11C).

\section{Discussion}

In the present study, we noted the following. Firstly, YAP1 was upregulated in HCC tumor tissues compared with adjacent tissues, and its high expression in tumors was associated with increased Edmonson grade in patients with HCC. Secondly, in vitro, YAP1 expression was increased, whereas that of pYAP1 was decreased, in HCC cell lines (Hep-3B, SK-HEP-1 and Huh7 cell lines) 
Hep-3B cells

A CK

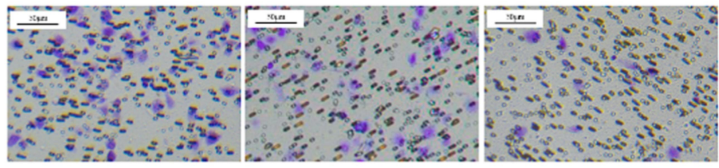

B

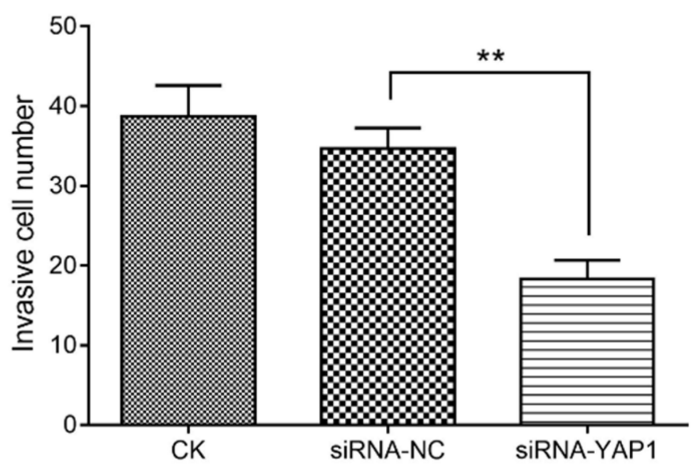

\section{SMMC-7721 cells}

C



D

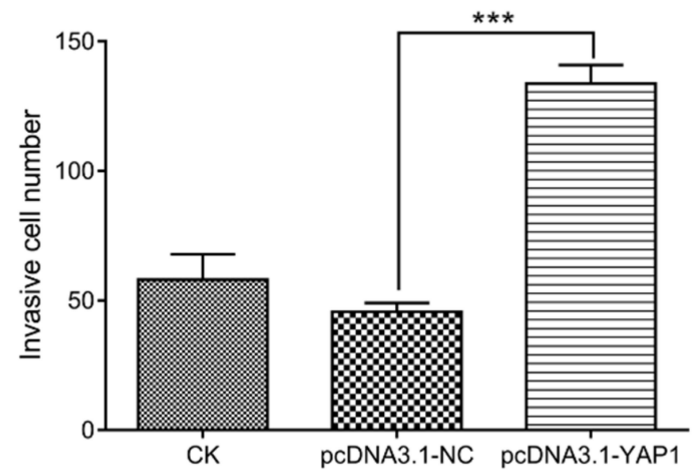

Figure 6 YAPI promoted cell invasion in HCC cells. Comparison of invasive cell number by Transwell assay between siRNA-YAPI recombinants transfected cells and siRNA-NC transfected cells in Hep-3B cells at $48 \mathrm{~h}$ after transfection (Aand B). Comparison of invasive cell number by Transwell assay between pcDNA3.I-YAPI plasmid transfected cells and pcDNA3.I-NC plasmid transfected cells in SMMC-772I cells at $48 \mathrm{~h}$ after transfection (Cand $\mathbf{D})$. $\mathrm{P}$ value was displayed as $* * \mathrm{P}<0.0 \mathrm{I}$ and $* * * \mathrm{P}<0.00 \mathrm{I}$. Abbreviations: YAPI, YesI associated transcriptional regulator; HCC, hepatocellular carcinoma; siRNA, small-interfering RNAs; NC, negative control; CK, control check.

compared with those detected in human normal liver cell line (LO2 cell line). YAP1 promoted cell proliferation, invasion, EMT progression, and the expression of its downstream gene Tead, but decreased cell apoptosis in HCC cells. Thirdly, in vivo, YAP1 knockdown exerted an inhibitory effect on tumor growth and metastasis, and demonstrated a synergistic anti-tumor effect with sorafenib in treating mice with $\mathrm{HCC}$, which was verified by cellular experiments.

YAP1, as a member of the YAP family, is a transcriptional coactivator which regulates tissue homeostasis and organ development by triggering numerous cell-autonomous responses, such as sustained proliferation, therapy resistance, cell plasticity, and metastasis. ${ }^{17}$ Accumulating published evidence exhibit the essential role of YAP1 as an oncogene in the initiation and progression of tumors via various ways. ${ }^{6,11,18}$ For example, a study indicated that YAP1 is upregulated in gastric cancer tissues compared with normal mucosal tissues, and its overexpression increases cell proliferation but decreases apoptosis. ${ }^{18}$ In another study, YAP1 expression was elevated in bladder cancer tissues compared with adjacent normal tissues, and its knockdown represses the proliferation ability and promotes apoptosis rate of bladder cancer cells. ${ }^{19}$ Clinically, high expression of YAP1 is associated with unfavorable tumor features in patients with various tumors, including gastric, non-small cell lung, colon, and breast cancer. $^{20-23}$ A study indicated that YAP1 is positively correlated with clinical stage, tumor size, and lymph node metastasis in patients with gastric cancer. ${ }^{20}$ Another study exhibited that high expression of YAP1 is correlated with poor differentiation, increased depth of invasion, and lymph node metastasis in patients with colon cancer. ${ }^{22}$ According to these previous findings, it was hypothesized that YAP1 may be correlated with clinicopathological features of patients with HCC. Confirming our hypothesis, we found that YAP1 was upregulated in HCC tumor tissues versus adjacent tissues, and high expression of YAP1 in tumors was associated with increased Edmonson grade in patients with HCC. The following possible reasons are considered. Firstly, according to the previous study, overexpression of YAP1 was positively related to the expression of the EMT transcriptional factors (eg, ZEB1), and the activation of EMT was involved in the HCC formation, during which inducing tumor-initiating epithelial cells undergo EMT to generate HCC. ${ }^{6}$ Therefore, YAP1 was 
Hep-3B cells

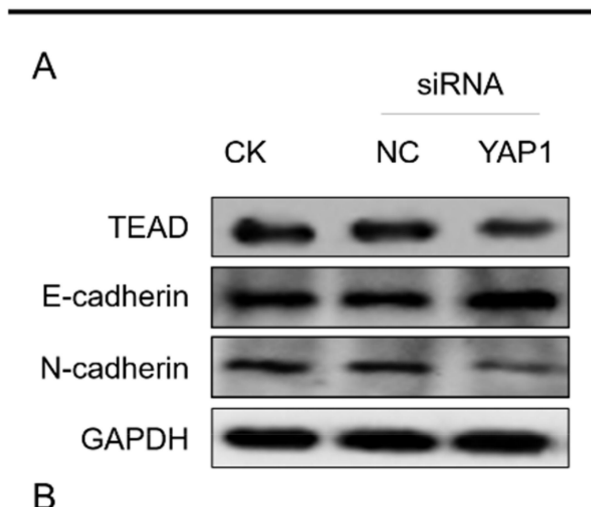

B

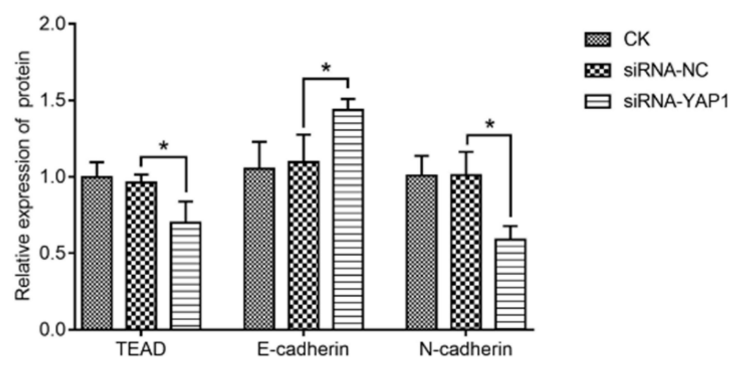

SMMC-7721 cells

C

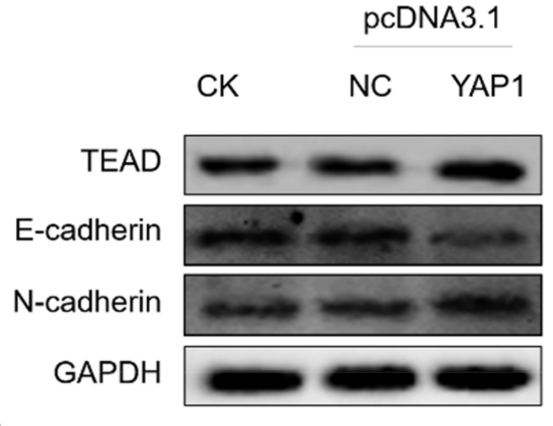

D

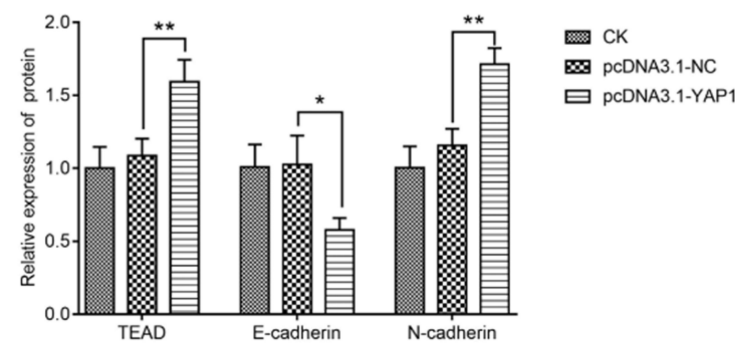

Figure 7 YAPI increased Tead expression and promoted EMT markers in HCC cells. Comparison of Tead, E-cadherin, and N-cadherin expression between Hep-3B cells transfected with siRNA-YAPI and those transfected with siRNA-NC (A and B), and between SMMC-772I cells transfected with the pcDNA3.I-YAPI plasmid and those transfected with the pcDNA3.I-NC plasmid (C and D). $P$ value was displayed as $* P<0.05$ and $* * P<0.01$.

Abbreviations: YAPI, YesI associated transcriptional regulator; HCC, hepatocellular carcinoma; siRNA, small-interfering RNAs; NC, negative control; EMT, epithelialmesenchymal transition; CK, control check.

upregulated in HCC tissues compared with adjacent tissues. Secondly, high expression of YAP1 was associated with increased stemness and EMT features, and the former may inhibit differentiation during tumor initiation and progression. Therefore, high expression of YAP1 was associated with poor tumor differentiation (increased Edmonson grade) in patients with HCC. ${ }^{6,24}$

Subsequently, we determined the expression of YAP1 in human HCC cell lines (Hep-3B, SMMC-7721, SK-HEP -1, and Huh-7) and a human normal liver cell line (LO2) through Western blotting. We found that YAP1 was upregulated in Hep-3B, SK-HEP-1, Huh-7 cell lines compared with LO2 cell line. We observed that YAP1 knockdown inhibited cell proliferation, invasion, whereas it promoted cell apoptosis in Hep-3B cells. In SMMC-7721 cells, overexpression of YAP1 promoted cell proliferation and invasion; however, the apoptosis rate presented a decreasing trend without statistical significance. This observation may be due to unobvious effect of YAP1 on cell apoptosis and relatively low apoptosis rate in SMMC-7721 cells. This hypothesis warrants further investigation. We also conducted cellular experiments to investigate the effect of
YAP1 overexpression on cell apoptosis in Hep-3B cells. The results indicated that overexpression of YAP1 inhibited cell apoptosis. These data suggest that YAP1 promoted the proliferation and invasion, whereas it inhibited the apoptosis of HCC cells. The results are consistent with those of a previous study reporting that silencing of YAP1 inhibited cell proliferation and invasion, but promoted apoptosis in thyroid cancer cells. ${ }^{25}$ In addition, we also found that YAP1 enhanced the expression of its downstream gene (Tead) in the Hippo signaling pathway in HCC cells, suggesting implication of this pathway in the pathology of the disease. However, this requires further exploration. Possible reasons are discussed below. Firstly, the Hippo signaling pathway is a tumor inhibitory signaling pathway, which medicates cell proliferation, apoptosis, invasion and migration. Hence, YAP1 may promote cell proliferation, invasion, migration, but inhibit apoptosis by acting with TEADs (which was verified in our present study) and inactivating Hippo signaling, which further leads to tumor growth and metastasis. ${ }^{6}$ Secondly, existing evidence indicates that EMT is a vital biological process involved in cancer invasion and metastasis, and prior data 


\section{Hep-3B cells}

A

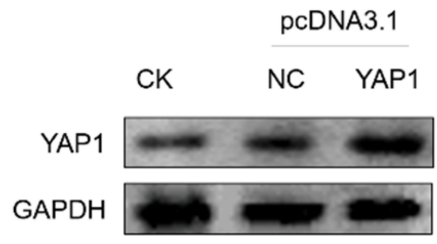

D

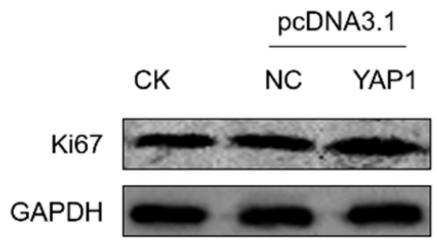

F



H

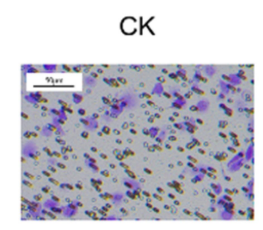

B



E

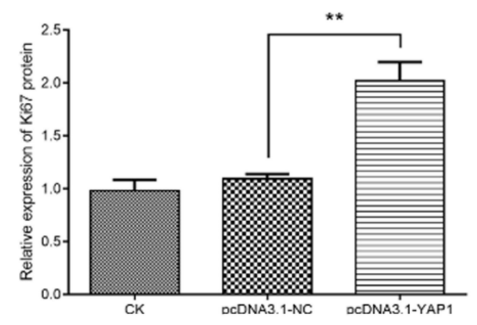

C

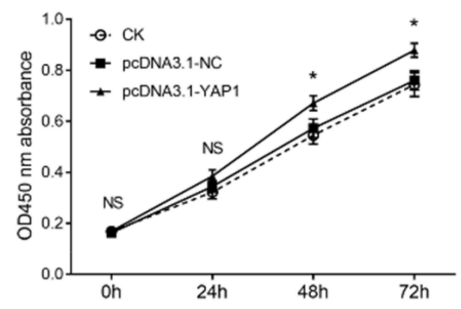

G

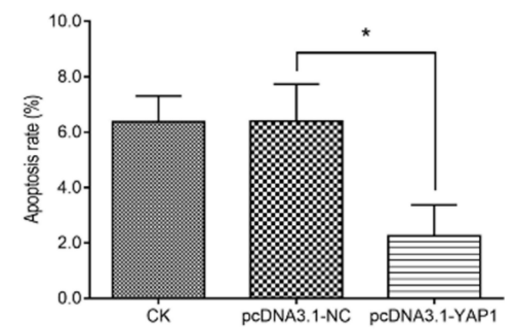

I

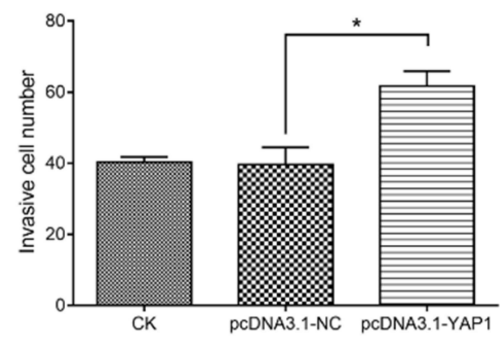

Figure 8 YAPI overexpression promoted proliferation and invasion, but inhibited apoptosis of Hep-3B cells. Comparison of YAPI expression (A and B), OD450 nm absorbance (C), Ki67 expression (D and E), apoptosis rate (F and $\mathbf{G})$ and number of invasive cells ( $\mathbf{H}$ and $\mathbf{I})$ between cells transfected with the pcDNA3.I-YAPI plasmid and

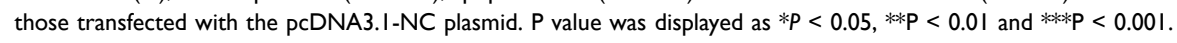

Abbreviations: YAPI, YesI associated transcriptional regulator; HCC, hepatocellular carcinoma; CCK-8, Cell Counting Kit-8; AV/PI, Annexin V/Propidium lodide; NC, negative control; CK, control check.

demonstrated that YAP1 enhanced the expression of $\mathrm{N}$-cadherin but decreased that of E-cadherin, further resulting to the increased invasion of $\mathrm{HCC}$ cells, ${ }^{26-28}$ These findings were verified in the current study. Thirdly, according to a previous study, activation of the tumor suppressor Hippo pathway led to cytoplasmic retention and further degradation of YAP1, while inactivation of the Hippo pathway contributed to the localization of YAP1 in the nucleus of HCC cells, as previously documented. ${ }^{29,30}$ Additionally, nuclear localization and 


\section{Hep-3B cells}

A

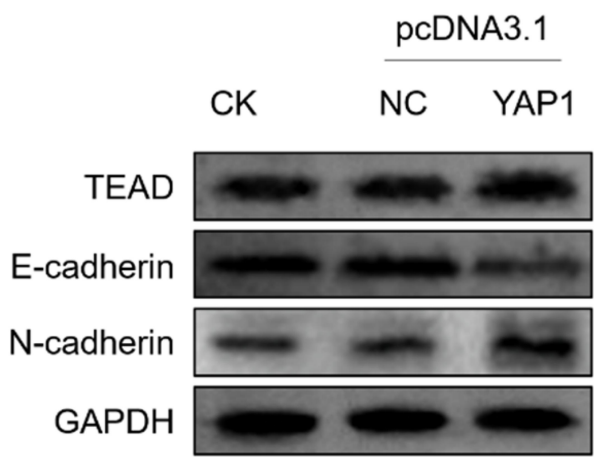

B

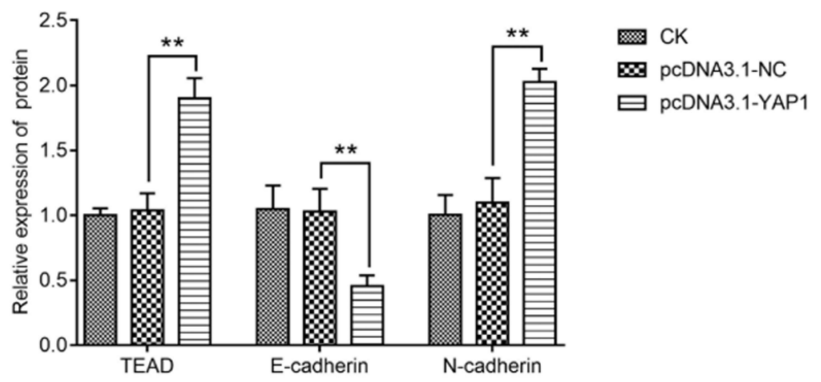

Figure 9 YAPI increased Tead expression and promoted EMT in Hep-3B cells. Comparison of Tead, E-cadherin, and N-cadherin expression (A and B) between cells transfected with the pcDNA3.I-YAPI plasmid and those transfected with the pcDNA3.I-NC plasmid. P value was displayed as **P < 0.01 .

Abbreviations: YAPI, YesI associated transcriptional regulator; HCC, hepatocellular carcinoma; siRNA, small-interfering RNAs; NC, negative control; EMT, epithelialmesenchymal transition; CK, control check.

A

B

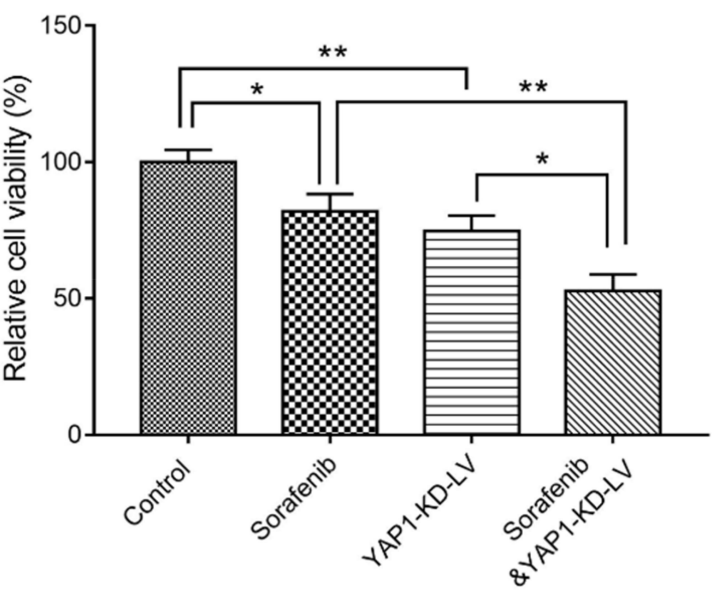

Control



Sorafenib

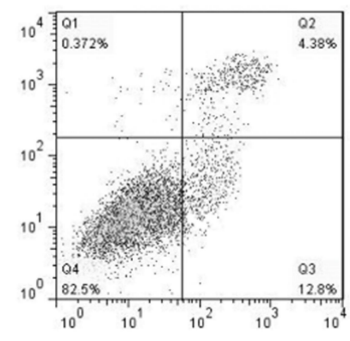

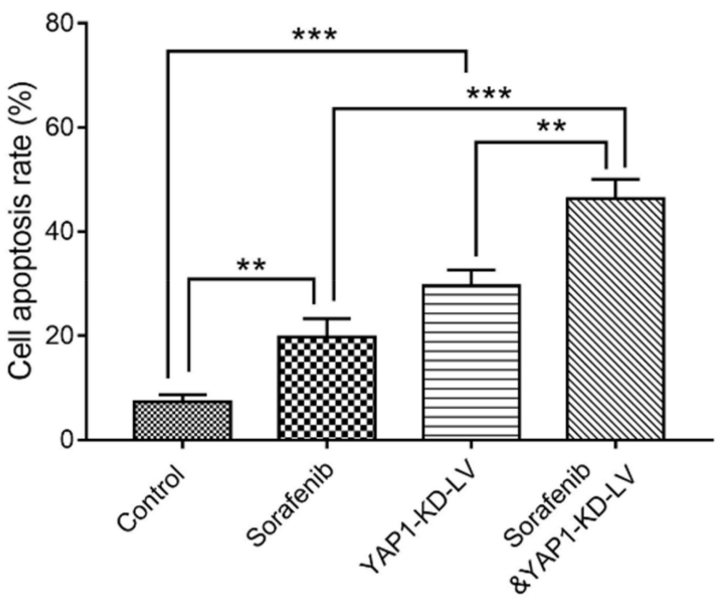

YAP1-KF-LV

Sorafenib \&YAP1-KD-LV
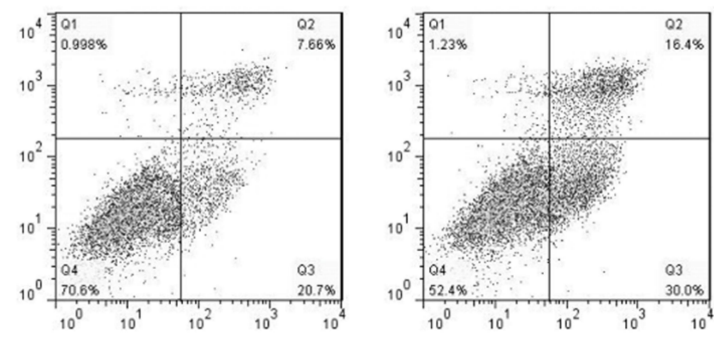

Annexin V-FITC

Figure 10 Combination of YAPI knockdown and administration of sorafenib inhibited cell viability, but promoted apoptosis of HCC cells. Comparison of relative cell viability $(\mathbf{A})$ and cell apoptosis rate (B and $\mathbf{C})$ among control cells, sorafenib cells, YAPI-KD-LV cells, and sorafenib and YAPI-KD-LV cells. P value was displayed as *P < 0.05 , **P $<0.01$ and *** $\mathrm{P}<0.001$.

Abbreviations: YAPI, YesI associated transcriptional regulator; HCC, hepatocellular carcinoma; YAPI-KD-LV, YAPI-knockdown lentivirus; NC-LV, negative control lentivirus. 
A

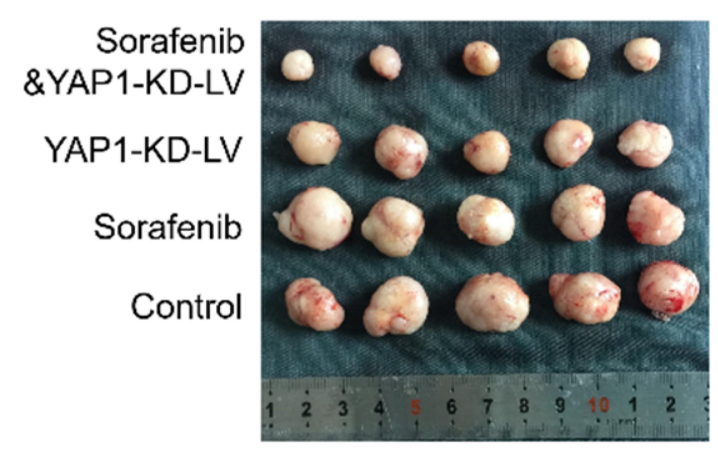

C
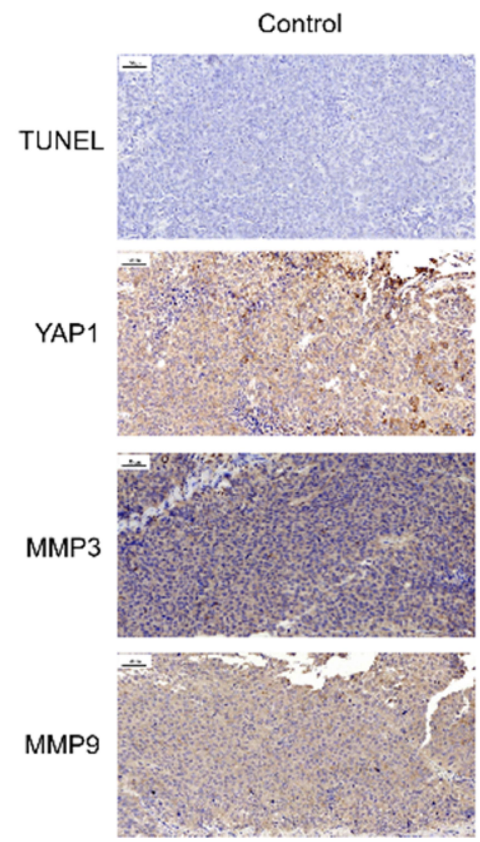

Sorafenib
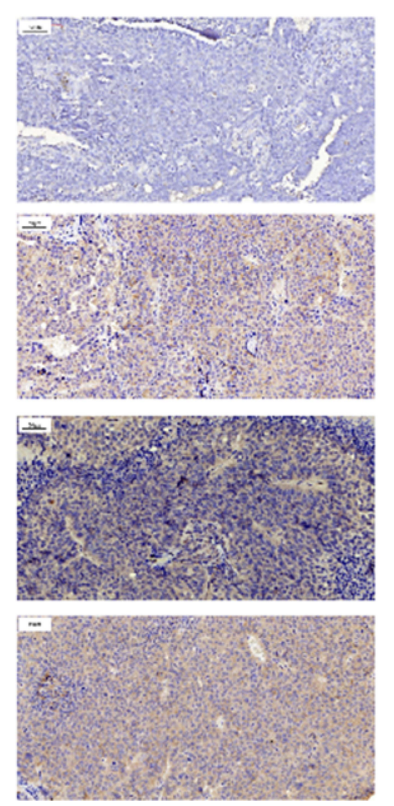

B

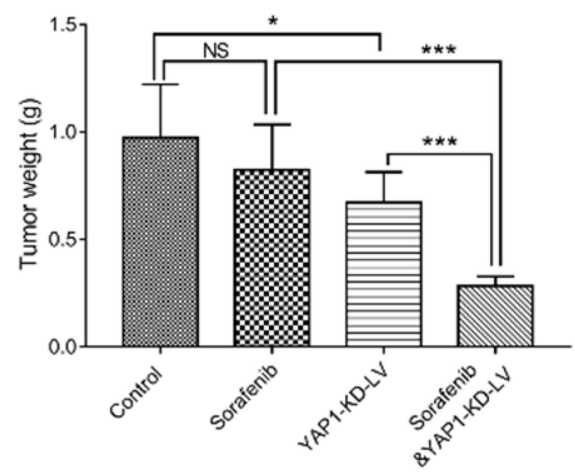

YAP1-KD-LV


Sorafenib\&YAP1-KD-LV
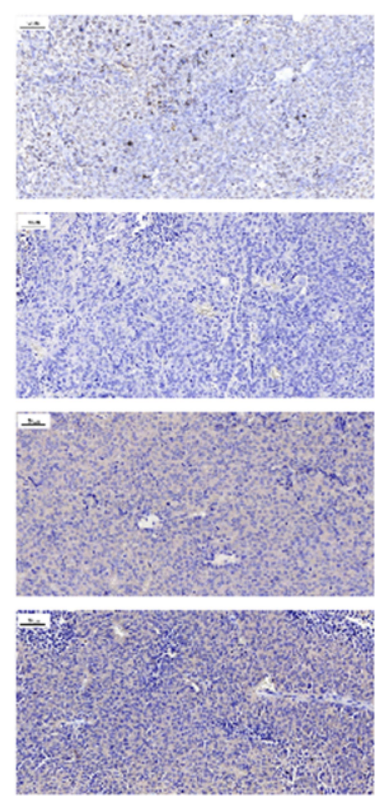

Figure I I Combination of YAPI knockdown and administration of sorafenib inhibited tumor growth in HCC. Comparison of tumor size (A) and tumor weight (B) among the Control, Sorafenib, YAPI-KD-LV, and Sorafenib \& YAPI-KD-LV groups. Comparison of tumor apoptosis, YAPI expression, MMP3 expression, and MMP9 expression among the Control, Sorafenib, YAPI-KD-LV, and Sorafenib \& YAPI-KD-LV groups (C). P value was displayed as $* P<0.05$, $* * * P<0.001$ and NS (P $>0.05$ ).

Abbreviations: YAPI, YesI associated transcriptional regulator; HCC, hepatocellular carcinoma; YAPI-KD-LV, YAPI-knockdown lentivirus; NC-LV, negative control lentivirus.

stabilization of YAP1 were correlated with increased HCC cell proliferation. ${ }^{31,32}$ Therefore, we hypothesized that YAP1 promoted cell proliferation and invasion, but inhibited cell apoptosis via nuclear localization and cytoplasmic degradation of YAP1. However, due to the limited budget, we could not cover the expense of additional cellular study to investigate the subcellular localization of YAP1 and related activity in HCC cells. This will be the focus of our future study.

Existing research studies demonstrated the association of YAP1 with the occurrence of intrinsic or acquired drug resistance in several types of tumors. In addition, accumulating data reveal that knockdown of YAP1 synergistically increases the efficacy of anti-tumor therapies, including molecular-targeted therapy, cytotoxic chemotherapy, and radiotherapy. ${ }^{6,11,33}$ For example, knockdown of YAP1 diminishes the resistance of HER2amplified breast cancer cells to lapatinib, and silencing of YAP1 in vivo attenuates the growth of implanted HER2-amplified tumors. ${ }^{34}$ In another study, overexpression of YAP1 increased the resistance of non-small cell lung cancer cells to erlotinib, and the combination of 
YAP1 inhibitor and erlotinib suppressed the tumorigenic properties of these cells. ${ }^{35}$ Furthermore, sorafenib is a multi-kinase inhibitor which possesses various antiproliferative, antiangiogenic, and proapoptotic properties, and is an effective first-line drug for the treatment of advanced HCC. ${ }^{36}$ According to the aforementioned evidence, we hypothesized that knockdown of YAP1 may synergize with sorafenib in the treatment of HCC; therefore, we performed the corresponding in vitro and in vivo experiments. We found that knockdown of YAP1 decrease HCC tumor growth. Of note, combined YAP1 knockdown and administration of sorafenib had some synergistic benefit on inhibiting tumor growth, which was also supported by the results of the cellular experiments. Furthermore, we performed the TUNEL assay and IHC assay. The results showed that the combination of YAP1 knockdown and sorafenib exerted a stronger effect on promoting apoptosis and inhibiting metastasis compared with YAP1 knockdown or treatment with sorafenib alone in HCC. These findings suggested that YAP1 knockdown synergized with sorafenib in the treatment of HCC. The possible reasons are presented below. Firstly, according to the previous studies, YAP1 is associated with the HCC stem cell phenotype, and its knockdown weakened the HCC stem cell properties. Hence, knockdown of YAP1 may have an inhibitory effect on tumor progression and synergize with sorafenib by suppressing stemness markers. ${ }^{37}$ Secondly, according to previous evidence, sorafenib served as an inhibitor of EGFR, and inactivation of EGFR signaling could suppress HCC cell growth via an YAP1-independent manner. Therefore, the combination of YAP1 knockdown and administration of sorafenib could exert a stronger inhibitory effect on tumor progression compared with YAP1 knockdown or sorafenib alone. ${ }^{38,39}$

\section{Conclusion}

YAP1 is upregulated in both HCC tumor tissues and cell lines, and promotes cell proliferation, invasion, and EMT progression in vitro. Meanwhile, targeting YAP1 inhibits HCC progression and improves sensitivity to sorafenib in vitro and in vivo. These data suggest that YAP1 may serve as a single or combined target in the treatment of HCC.

\section{Abbreviations}

YAP1, Yes1 associated transcriptional regulator; HCC, hepatocellular carcinoma; EGFR, epidermal growth factor receptor; HRP, horseradish peroxidase; GAPDH, glyceraldehyde-3-phosphate dehydrogenase; siRNA, smallinterfering RNAs; siRNA-NC, non-targeting siRNA; pYAP1, phosphorylated YAP1; CK, control check; FIRC, fluorescein isothiocyanate; $\mathrm{NC}$, negative control; OD, optical density; CCK-8, Cell Counting Kit-8; AV/PI, Annexin V/propidium iodide; MMP, matrix metalloproteinase; EMT, epithelial-mesenchymal transition; YAP1-KD-LV, YAP1-knockdown lentivirus; NC-LV, negative control lentivirus; CI, combination index.

\section{Data Sharing Statement}

The datasets used and/or analysed during the current study are available from the corresponding author on reasonable request.

\section{Ethics Approval and Consent to Participate}

The animal study was approved by the Institutional Animal Care and Use Committee of Cancer Hospital of the University of Chinese Academy of Sciences (Hangzhou, China), following the Guidelines for the ethical review of laboratory animal welfare People's Republic of China National Standard GB/T35892-2018.

\section{Funding}

This study was funded by Medical Science and Technology Planning Project of Zhejiang Province (Project No: 2018RC023), Zhejiang province key research project (Project No:2019C03058).

\section{Disclosure}

The authors report no conflicts of interest in this work.

Liwen Guo and Jiaping Zheng are co-first authors for this study.

\section{References}

1. Heimbach JK, Kulik LM, Finn RS, et al. AASLD guidelines for the treatment of hepatocellular carcinoma. Hepatology. 2018;67 (1):358-380.

2. Wallace MC, Preen D, Jeffrey GP, Adams LA. The evolving epidemiology of hepatocellular carcinoma: a global perspective. Expert Rev Gastroenterol Hepatol. 2015;9(6):765-779. doi:10.1586/ 17474124.2015.1028363

3. Vogel A, Cervantes A, Chau I, et al. Hepatocellular carcinoma: ESMO clinical practice guidelines for diagnosis, treatment and follow-up. Ann Oncol. 2019;30(5):871-873. doi:10.1093/annonc/mdy510

4. Rinninella E, Cerrito L, Spinelli I, et al. Chemotherapy for hepatocellular carcinoma: current evidence and future perspectives. J Clin Transl Hepatol. 2017;5(3):235-248. 
5. Kinoshita A, Onoda H, Fushiya N, Koike K, Nishino H, Tajiri H. Staging systems for hepatocellular carcinoma: current status and future perspectives. World $J$ Hepatol. 2015;7(3):406-424. doi:10.4254/wjh.v7.i3.406

6. Shibata M, Ham K, Hoque MO. A time for YAP1: tumorigenesis, immunosuppression and targeted therapy. Int $J$ Cancer. 2018;143 (9):2133-2144. doi:10.1002/ijc.31561

7. Zhao B, Lei QY, Guan KL. The Hippo-YAP pathway: new connections between regulation of organ size and cancer. Curr Opin Cell Biol. 2008;20(6):638-646.

8. Cheng H, Zhang Z, Rodriguez-Barrueco R, et al. Functional genomics screen identifies YAP1 as a key determinant to enhance treatment sensitivity in lung cancer cells. Oncotarget. 2016;7 (20):28976-28988. doi:10.18632/oncotarget.6721

9. Shen J, Cao B, Wang Y, et al. Hippo component YAP promotes focal adhesion and tumour aggressiveness via transcriptionally activating THBS1/FAK signalling in breast cancer. J Exp Clin Cancer Res. 2018;37(1):175. doi:10.1186/s13046-018-0850-Z

10. Andrade D, Mehta M, Griffith J, et al. YAP1 inhibition radiosensitizes triple negative breast cancer cells by targeting the DNA damage response and cell survival pathways. Oncotarget. 2017;8 (58):98495-98508. doi:10.18632/oncotarget.21913

11. Lu T, Sun L, Zhu X. Yes-associated protein enhances proliferation and attenuates sensitivity to cisplatin in human gastric cancer cells. Biomed Pharmacother. 2018;105:1269-1275. doi:10.1016/j. biopha.2018.06.031

12. Sun D, Li X, He Y, et al. YAP1 enhances cell proliferation, migration, and invasion of gastric cancer in vitro and in vivo. Oncotarget. 2016;7(49):81062-81076. doi:10.18632/oncotarget.13188

13. Li P, Sun D, Li X, et al. Elevated expression of Nodal and YAP1 is associated with poor prognosis of gastric adenocarcinoma. $J$ Cancer Res Clin Oncol. 2016;142(8):1765-1773. doi:10.1007/s00432-0162188-2

14. Wang X, Wu B, Zhong Z. Downregulation of YAP inhibits proliferation, invasion and increases cisplatin sensitivity in human hepatocellular carcinoma cells. Oncol Lett. 2018;16(1):585-593.

15. Guidelines for the ethical review of laboratory animal welfare. GB/ T35892-2018. Standardization administration of China Beijing ICP 09001239. Available from: http://www.gb688.cn/bzgk/gb/ newGbInfo?hcno=9BA619057D5C13103622A10FF4BA5D14.

16. Chou TC. Drug combination studies and their synergy quantification using the Chou-Talalay method. Cancer Res. 2010;70(2):440-446. doi:10.1158/0008-5472.CAN-09-1947

17. Zanconato F, Cordenonsi M, Piccolo S. YAP and TAZ: a signalling hub of the tumour microenvironment. Nat Rev Cancer. 2019;19 (8):454-464. doi:10.1038/s41568-019-0168-y

18. Du F, Yu C, Li R, Ding D, He L, Wen G. Expression of miR-141 and YAP1 in gastric carcinoma and modulation of cancer cell proliferation and apoptosis. Int J Clin Exp Pathol. 2019;12(2):559-567.

19. Xu M, Gu M, Zhou J, Da J, Wang Z. Interaction of YAP1 and mTOR promotes bladder cancer progression. Int $J$ Oncol. 2020;56 (1):232-242.

20. Huang S, Zhu L, Cao Y, et al. Significant association of YAP1 and HSPC111 proteins with poor prognosis in Chinese gastric cancer patients. Oncotarget. 2017;8(46):80303-80314. doi:10.18632/ oncotarget.17932

21. Chen MJ, Wang YC, Wu DW, Chen CY, Lee H. Association of nuclear localization of SHP2 and YAP1 with unfavorable prognosis in non-small cell lung cancer. Pathol Res Pract. 2019;215 (4):801-806. doi:10.1016/j.prp.2019.01.027

22. Shao Q, Xu J, Deng R, et al. The expressions of YAP1, beta-catenin and survivin in colon cancer tissues and their clinical significance. Int J Clin Exp Pathol. 2018;11(12):6032-6038.
23. Yu S, Zhang M, Huang L, et al. ERK1 indicates good prognosis and inhibits breast cancer progression by suppressing YAP1 signaling. Aging (Albany NY). 2019;11(24):12295-12314. doi:10.18632/ aging. 102572

24. Jin X, Jin X, Kim H. Cancer stem cells and differentiation therapy. Tumour Biol. 2017;39(10):1010428317729933. doi:10.1177/ 1010428317729933

25. Li D, Wang Q, Li N, Zhang S. miR205 targets YAP1 and inhibits proliferation and invasion in thyroid cancer cells. Mol Med Rep. 2018;18(2):1674-1681.

26. Zhou Y, Yang R, Ma G. [YAP1 knockdown suppresses the proliferation, migration and invasion of human nasopharyngeal carcinoma cells]. Nan Fang Yi Ke Da Xue Xue Bao. 2019;39(3):286-291.

27. Kaowinn S, Yawut N, Koh SS, Chung YH. Cancer upregulated gene (CUG)2 elevates YAP1 expression, leading to enhancement of epithelial-mesenchymal transition in human lung cancer cells. Biochem Biophys Res Commun. 2019;511(1):122-128. doi:10.1016/ j.bbrc.2019.02.036

28. Sun Z, Ou C, Liu J, et al. YAP1-induced MALAT1 promotes epithelial-mesenchymal transition and angiogenesis by sponging miR-126-5p in colorectal cancer. Oncogene. 2019;38 (14):2627-2644. doi:10.1038/s41388-018-0628-y

29. LaQuaglia MJ, Grijalva JL, Mueller KA, et al. YAP subcellular localization and Hippo pathway transcriptome analysis in pediatric hepatocellular carcinoma. Sci Rep. 2016;6:30238.

30. Dong Q, Fu L, Zhao Y, Xie C, Li Q, Wang E. TNFAIP8 interacts with LATS1 and promotes aggressiveness through regulation of Hippo pathway in hepatocellular carcinoma. Oncotarget. 2017;8 (9):15689-15703. doi:10.18632/oncotarget.14938

31. Larsen S, Davidsen J, Dahlgaard K, Pedersen OB, Troelsen JT. HNF4 $\alpha$ and CDX2 regulate intestinal YAP1 promoter activity. Int J Mol Sci. 2019;20(12):2981. doi:10.3390/ijms20122981

32. Zhang X, Li Y, Ma Y, et al. Yes-associated protein (YAP) binds to HIF-1 $\alpha$ and sustains HIF-1 $\alpha$ protein stability to promote hepatocellular carcinoma cell glycolysis under hypoxic stress. J Exp Clin Cancer Res. 2018;37(1):216. doi:10.1186/s13046-018-0892-2

33. Kim MH, Kim J. Role of YAP/TAZ transcriptional regulators in resistance to anti-cancer therapies. Cell Mol Life Sci. 2017;74 (8):1457-1474. doi:10.1007/s00018-016-2412-x

34. Lin $\mathrm{CH}$, Pelissier FA, Zhang $\mathrm{H}$, et al. Microenvironment rigidity modulates responses to the HER2 receptor tyrosine kinase inhibitor lapatinib via YAP and TAZ transcription factors. Mol Biol Cell. 2015;26(22):3946-3953. doi:10.1091/mbc.E15-07-0456

35. Hsu PC, You B, Yang YL, et al. YAP promotes erlotinib resistance in human non-small cell lung cancer cells. Oncotarget. 2016;7 (32):51922-51933. doi:10.18632/oncotarget.10458

36. Mendez-Blanco C, Fondevila F, Garcia-Palomo A, GonzalezGallego J, Mauriz JL. Sorafenib resistance in hepatocarcinoma: role of hypoxia-inducible factors. Exp Mol Med. 2018;50(10):134. doi:10.1038/s12276-018-0159-1

37. Kim GJ, Kim H, Park YN. Increased expression of Yes-associated protein 1 in hepatocellular carcinoma with stemness and combined hepatocellular-cholangiocarcinoma. PLoS One. 2013;8(9):e75449. doi:10.1371/journal.pone.0075449

38. Dai N, Ye R, He Q, Guo P, Chen H, Zhang Q. Capsaicin and sorafenib combination treatment exerts synergistic antihepatocellular carcinoma activity by suppressing EGFR and PI3K/Akt/mTOR signaling. Oncol Rep. 2018;40(6):3235-3248.

39. Lee TF, Tseng YC, Chang WC, et al. YAP1 is essential for tumor growth and is a potential therapeutic target for EGFR-dependent lung adenocarcinomas. Oncotarget. 2017;8(52):89539-89551. doi:10.18632/oncotarget.19647 


\section{Publish your work in this journal}

OncoTargets and Therapy is an international, peer-reviewed, open access journal focusing on the pathological basis of all cancers, potential targets for therapy and treatment protocols employed to improve the management of cancer patients. The journal also focuses on the impact of management programs and new therapeutic agents and protocols on patient perspectives such as quality of life, adherence and satisfaction. The manuscript management system is completely online and includes a very quick and fair peer-review system, which is all easy to use. Visit http://www.dovepress.com/ testimonials.php to read real quotes from published authors. 